\title{
Crenças, opiniões e atitudes dos enfermeiros de saúde mental, face à doença psiquiátrica
}

Creencias, opiniones y actitudes de los enfermeros de salud mental frente a la enfermedad psiquiátrica Beliefs, Opinions and Attitudes of Mental Health Nurses Towards Psychiatric Disease

\author{
Paula Campos ${ }^{1}$, José Carlos Carvalho² $^{2}$ Júlia Martinho² \\ 'UCC Castelo da Maia, ACES Maia/Valongo. Maia, Portugal. \\ ${ }^{2}$ Escola Superior de Enfermagem do Porto, Portugal/Center for Health Technology and Services Research (CINTESIS). Porto, Portugal. \\ Contacto: zecarlos@esenf.pt \\ Fecha de recepción: 22 de octubre de 2018 / Fecha de aceptación: 22 de noviembre de 2018
}

\begin{abstract}
Resumo
A ação do estigma sobre as pessoas com patologia psiquiátrica tem como consequência a discriminação nos cuidados e na vida quotidiana, priva-os do exercício de direitos como a autonomia e a da liberdade e exclui-os de toda e qualquer participação no projeto de vida.

Investigação com um desenho quantitativo, descritivo e correlacional, tendo como objetivos, analisar de que forma as crenças, opiniões e atitudes dos enfermeiros de saúde mental contribuem para a redução/aumento do estigma na doença mental e descrever e comparar as opiniões e atitudes dos profissionais em função das caraterísticas sociodemográficas. Utilizados como instrumentos de avaliação: questionário sociodemográfico, a Escala de Opiniões acerca da Doença Mental, o Inventário de Crenças sobre a doença Mental (ICDM) e Escala das Atitudes dos Clínicos (MICA-4). Amostra de 93 enfermeiros que desempenham funções área da saúde mental em vários serviços da região norte de Portugal.

Os resultados demonstram, que existe pouca influência dos dados demográficos, pessoais e académicos nas crenças, opiniões e atitudes dos profissionais face à doença mental, assim como a opinião e as crenças parecem ter uma relação direta com as atitudes e com a presença de estigma.

Os enfermeiros não são agentes estigmatizantes; a formação específica funciona como elemento protetor contra o estigma. Parece existir uma relação entre a opinião e as crenças dos inquiridos sobre a doença mental e com a presença de estigma. Seria importante, trabalhar a cidadania, a inclusão e a patologia psiquiátrica, à semelhança dos programas implementados nas escolas junto dos mais jovens.
\end{abstract}

Palavras-chave: doença mental, enfermeiros, crenças, opiniões, atitudes, estigma.

\section{Resumen}

La acción del estigma sobre las personas con patología psiquiátrica tiene como consecuencia la discriminación en los cuidados y en la vida cotidiana, los priva del ejercicio de derechos como la autonomía y la libertad y los excluye de toda participación en el proyecto de vida.

Investigación con un diseño cuantitativo, descriptivo y correlacional, teniendo como objetivos, analizar de qué forma las creencias, opiniones y actitudes de los enfermeros de salud mental contribuyen a la reducción/aumento del estigma en la enfermedad mental y describir y comparar las opiniones y actitudes de los profesionales en función de las características sociodemográficas.

Se utilizaron como instrumentos de evaluación: cuestionario sociodemográfico, la Escala de opiniones sobre la enfermedad mental, el inventario de creencias sobre la enfermedad mental (ICEM) y la escala de las actitudes de los médicos (MICA-4). Muestra de 93 enfermeras que realizan las funciones de campo de la salud mental en diversos servicios en la región norte de Portugal.

Palabras clave: enfermedad mental, enfermeras, creencias, opiniones, actitudes, estigma.

\section{Abstract}

The action of stigma on people with psychiatric disorders results in discrimination in care and daily life, deprives them of the exercise of rights such as autonomy and freedom, and excludes them from any participation in the project of life. 
Research with a quantitative, descriptive and correlational design, aiming to analyze how the beliefs, opinions and attitudes of mental health nurses contribute to the reduction / increase of stigma in mental illness and to describe and compare the opinions and attitudes of professionals according to sociodemographic characteristics.

Used as evaluation instruments: sociodemographic questionnaire, Mental Illness Opinions Scale, Mental Illusions Belief Inventory (MIBI) and Clinical Attitudes Scale (MICA-4). Sample of 93 nurses who perform mental health area functions in several services in the northern region of Portugal.

The results show that there is little influence of demographic, personal and academic data on professionals' beliefs, opinions and attitudes towards mental illness, just as opinion and beliefs seem to have a direct relationship with attitudes and the presence of stigma.

Nurses are not stigmatizing agents; specific training works as a protective element against stigma. There seems to be a relationship between opinion and beliefs of respondents about mental illness and the presence of stigma. It would be important to work on citizenship, inclusion and psychiatric pathology, much like the programs implemented in schools with the younger ones.

Keywords: mental illness, nurses, beliefs, opinions, attitudes, stigma.

\section{Introdução}

Este estudo surgiu no âmbito do Mestrado em Enfermagem de saúde Mental e Psiquiatria e é parte integrante do projeto "Representações, famílias e modelos de intervenção em saúde - REFAMIS" que é um dos projetos da unidade pedagógica Enfermagem, Disciplina e Profissão da ESEP e tem como objetivo estudar as "opiniões - crenças" de estudantes universitários, profissionais de saúde, pacientes, famílias e público em geral sobre doenças alvo de estigma, a fim de aumentar os níveis de conhecimento e literacia em saúde, reduzindo as atitudes e as repercussões negativas e consequentemente promover comportamentos mais inclusivos que respondam às necessidades específicas destas populações.

No exercício da minha atividade como enfermeira, deparo-me muitas vezes, com a dificuldade dos doentes mentais em assumirem a sua patologia, contrariamente a doentes portadores de outro tipo de patologias. Esta necessidade de ocultarem ou mesmo não assumirem, prende-se com o receio de serem rotulados, de serem descriminados. Consequentemente, estas defesas perante a patologia e perante a opinião dos demais, atrasa a procura de ajuda, prejudicando significativamente a atividade terapêutica, a recuperação e em alguns casos a reinserção social.

Ao realizar-se uma pesquisa sobre o tema, deparamo-nos com alguns estudos a comprovar a existência de estigma relacionado com a doença mental, nos quais os profissionais de saúde são implicados, o que causa alguma preocupação, pelo carácter educativo e forma- tivo destes profissionais nos cuidados. Assim, senti ser pertinente, estudar a população de enfermeiros que exercem funções em serviços de psiquiatria. Perceber de que forma as suas crenças, opiniões e atitudes face à doença mental, contribuem ou não para o aumento do estigma para com os doentes psiquiátricos.

Para melhor compreensão foi necessário neste estudo definir conceitos que suportam a temática do estigma, como é o caso de: Saúde e Saúde Mental; Doença e Doença Mental; Crença, Opinião e Atitude; Estigma; Preconceito e Descriminação

Neste artigo selecionamos três conceitos, que são os que os instrumentos de colheita de dados por nos escolhidos, avaliam.

O termo Crença (derivado do latim "credere" que em português significa "crer"), sinónimo de convicção, designa uma disposição para admitir ou aceitar algo, para aderir a uma opinião, ou a uma ideologia. $\mathrm{O}$ preconceito, a ingenuidade, a opinião e até mesmo o saber e a fé são diferentes formas de crença. ${ }^{1}$

A opinião provem do latim "opinio", significa uma ideia confusa acerca da realidade e que se opõe ao conhecimento. No sentido corrente, opinião é um ponto de vista pessoal a respeito de algo, que consideramos verdadeiro precisamente porque é nosso. A opinião é um enunciado que se pretende seja verdadeiro. As opiniões são emitidas de forma espontânea e direta, não necessitando de definições, explicações, justificações e argumentações. ${ }^{2}$ 
Um dos conceitos mais importante da psicologia social tem sido o de Atitude. As atitudes, são "as convicções e sentimentos que predispõem as nossas reações a objetos, pessoas e acontecimentos" se acreditarmos que alguém "é má pessoa e tem mau carácter", podemos sentir aversão por ela e agir com hostilidade. ${ }^{3}$ Para o mesmo autor, as atitudes refletem o modo como pensamos e nos sentimos em relação às pessoas, objetos e questões do meio em que nos inserimos. ${ }^{4}$

As atitudes e comportamentos das pessoas em relação a determinados assuntos, como são exemplo: a religião e minorias raciais, revelaram que é comum pensar de forma diferente da que agimos. Essa aparente discrepância surpreendeu os psicólogos sociais, a maioria dos quais partilhava a convicção de que existia uma relação estreita entre o pensamento e a ação, carácter e a conduta, as palavras particulares e os efeitos públicos. As pressões sociais podem encobrir a ligação latente entre as nossas atitudes e as respetivas ações, afetando o que dizemos ou fazemos. Quando conscientes de que as nossas atitudes e ações não coincidem, o indivíduo experimenta o sentimento de tensão interior, o qual os psicólogos denominam de dissonância cognitiva.

\section{Problematização do estigma na doença mental}

Contextualizado a problemática do estigma em saúde mental, este representa geralmente uma perceção negativa da pessoa com patologia psiquiátrica, presumindo-se que são diferentes dos demais, o que implica uma desvalorização e descriminação do seu papel na sociedade. O não reconhecimento dos sintomas e doença, mais o medo da rotulagem, inibe o processo de aceitação do seu estado de doença, bem como a participação esclarecida nos cuidados. ${ }^{5-7}$

\section{Metodologia}

Quanto à metodologia, e após termos delimitado o problema, procedemos à pesquisa bibliográfica que nos permitiu traçar os objetivos deste estudo e a escolha do desenho de investigação. Assim, a investigação que nos propomos, obedece a um desenho característico descritivo correlacional, uma vez que pretendemos descrever o universo de uma determinada população ou fenómeno e estabelecer relação entre variáveis, ou seja, descrever as opiniões e atitudes que os enfermeiros apresentam face à doença mental e psiquiátrica $\mathrm{e}$ relacioná-las com um conjunto de caraterísticas sociodemográficas e de contexto.

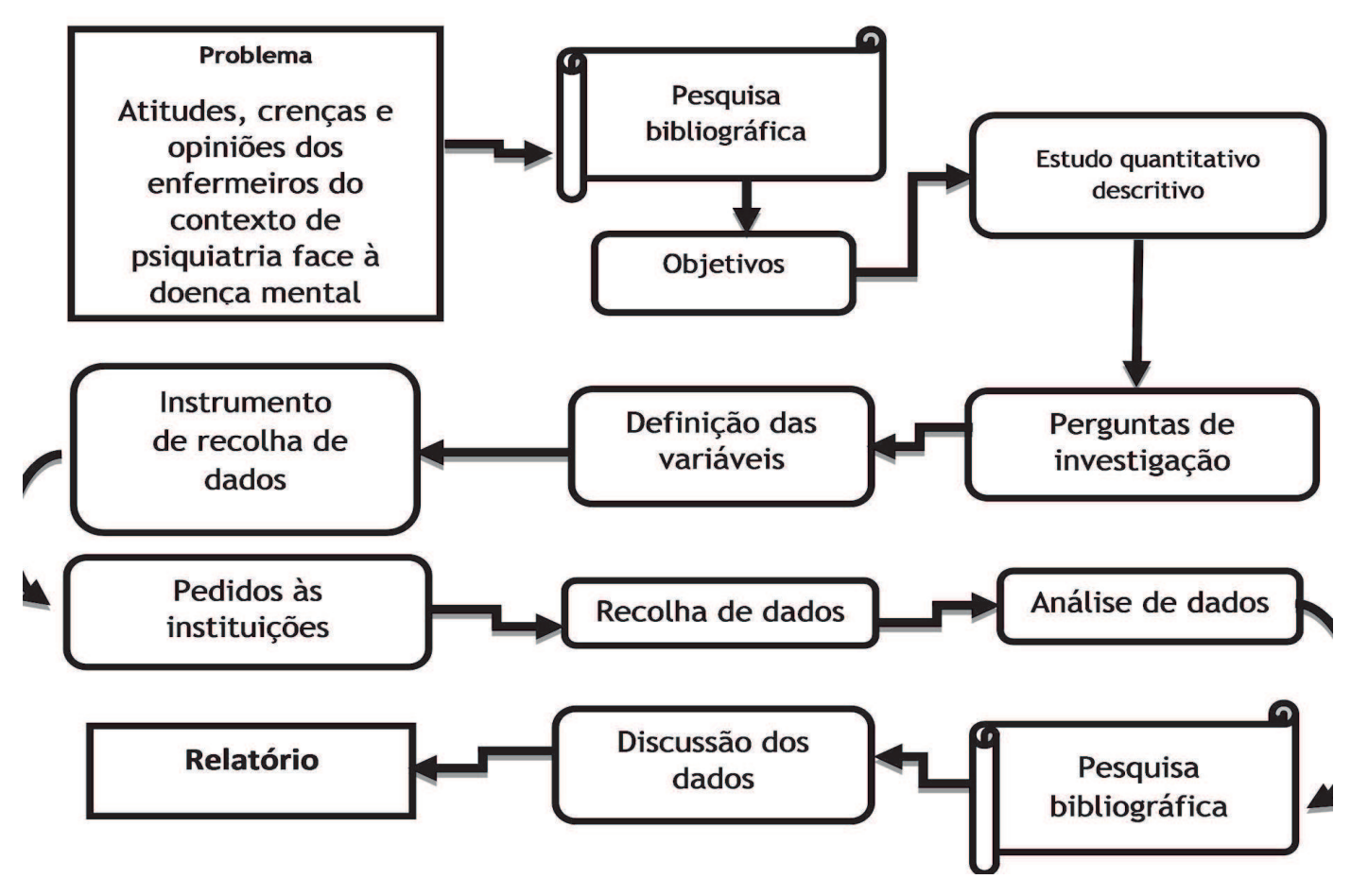

Figura 1. Fluxograma da investigação 
É um estudo transversal porque iremos avaliar a frequência de um determinado fenómeno numa determinada população, mais especificamente, a frequência de atitudes estigmatizantes na população de enfermeiros de saúde mental. Optamos por um estudo quantitativo, porque esta investigação apresenta como objetivos, a identificação e apresentação de dados, indicadores e tendências observáveis.

Esclarecidas as questões de investigação, definimos as variáveis em estudo e quais os instrumentos que melhor de adequavam à sua avaliação. Posteriormente, selecionamos a nossa amostra e solicitamos autorizações aos Conselhos de administração e Comissões de Ética. Após as autorizações de CES, procedemos a distribuição dos questionários, pelos serviços e posteriormente a sua recolha. Os dados foram analisados e novamente se procedeu à pesquisa bibliográfica, no sentido de encontrar estudos cujos resultados pudessem ser comparados com os obtidos no nosso estudo.

\section{Objetivos}

Assim, o objetivo do nosso estudo surge com a necessidade de entender e analisar de que forma as crenças, opiniões e atitudes dos enfermeiros de saúde mental contribuem para a redução ou aumento do estigma na doença mental.

\section{População e amostra}

Da população de 307 enfermeiros, foi possivel contar com a participação de 93 enfermeiros com os questionórios preenchidos na totalidade,

Como critérios de inclusão no estudo definimos: Enfermeiros que exercem funções em serviços de internamento de psiquiatria em instituições hospitalares na região norte; aceitar participar no estudo conhecendo os objetivos e finalidade do mesmo. Como critérios de exclusão consideramos: A recusa na participação no estudo e o preenchimento incompleto ou incorreto dos questionários.

\section{Instrumentos de colheita de dados}

A caracterização sociodemográfica, foi obtida através de um questionário com 14 questões de resposta fechada e aberta, relativas às características pessoais e profissionais dos enfermeiros sendo que uma das perguntas procura saber se os enfermeiros têm famili- ares ou amigos com patologia psiquiátrica.

Para se perceber quais as opiniões face à doença mental, foi utilizada a versão portuguesa de Oliveira (2005) da Escala de Opiniões acerca da Doença Mental, é uma escala tipo Likert, composta por 51 itens, cujas respostas variam entre o 1 (concordo completamente) e o 6 (discordo completamente). Organizada e subdividida em 5 fatores que correspondem a 5 atitudes:

- Autoritarismo (onde se pretende avaliar a opinião do inquirido, sobre se o sujeito com patologia psiquiátrica pertence a uma "classe de pessoas com valor inferior");

- Benevolência (pretende avaliar se o paternalismo do inquirido em relação ao individuo com patologia psiquiátrica, esta presente nas suas respostas);

- Ideologia da Higiene Mental (pretende-se perceber se o inquirido tem uma perceção do doente mental como sendo uma "pessoa normal");

- Restrição Social (perceber se o inquirido vê no doente mental, perigosidade para a sociedade);

- Etiologia Interpessoal (perceber a opinião do inquirido quanto à crença de que a doença mental é resultante das más $\mathrm{s}$ experiencias interpessoais vividas na infância).

Para a análise das crenças sobre a doença mental, utilizamos o Inventário de Crenças sobre a doença Mental (ICDM), validado para a população portuguesa por Loureiro (2009). É uma escala tipo Likert, composta por 47 itens, distribuídos pelos seguintes fatores: traumas emocionais e profissionais; traumas à nascença; doença e comportamentos de risco; causas associadas ao envelhecimento e deterioração. $\mathrm{O}$ formato da resposta varia entre 1 (discordo completamente) e 6 (concordo totalmente).

Foi igualmente utilizado uma Escala das Atitudes dos Clínicos (profissionais de saúde) (MICA-4), diz respeito às afirmações sobre as atitudes dos participantes face à doença mental e à psiquiatria, a qual é constituída por 16 itens. ${ }^{8}$

É também uma escala de tipo Likert, com respostas que vão desde o 1 (concordo plenamente) ao 6 (discordo totalmente). ${ }^{9}$

A cotação é realizada pela soma dos valores correspondentes a cada resposta, formulados em sentido inverso, o que significa que são cotados de forma 
invertida, ou seja, em que o valor 6 corresponde ao discordo totalmente e o valor 1 ao concordo totalmente, sendo que resultados mais baixos representam perceções menos estigmatizantes face à psiquiatria e à doença mental, com uma amplitude de [16/96]. Este instrumento foi alvo de tradução, adaptação e validação para a população portuguesa no âmbito do projeto "Representações, Famílias e Modelos de Intervenção em Saúde”.

\section{Considerações éticas}

Foram respeitados os princípios éticos, a participação no estudo foi voluntária, tendo por base um consentimento informado e presumido, anexado ao pedido de colaboração dos participantes; ficaram asseguradas a confidencialidade e anonimato pela codificação dos questionários (não sendo passível de identificar instituição, serviço e/ou participante), bem como a possibilidade de desistência ou não colaboração no projeto. Não houve qualquer tipo de financiamento, tendo os custos financeiros inerentes à realização do estudo sido financiados pelo investigador.

\section{Resultados}

No que diz respeito à caracterização estatística da amostra, e como já foi referido anteriormente, a amostra do presente estudo ficou constituída por 93 profissionais de saúde que desempenham funções na área da saúde mental. Os inquiridos apresentam idades compreendidas entre os 20 e os 59 anos, sendo que em média a amostra apresenta 39 anos de idade $(\mathrm{m}=38,8$; $\mathrm{dp}=10,26$ ). A percentagem mais expressiva de elementos pertence ao sexo feminino (mais propriamente $66,7 \%, \mathrm{n}=62$ ).
A amostra é composta maioritariamente por elementos casados $(59,85, n=55)$ e que trabalha na área do internamento $(92,9 \% ; n=79)$.

Inventário de Crenças sobre a doença mental (ICDM), composto por seis dimensões.

Manifestam uma crença moderada baixa da incurabilidade e incapacidade crónica dos doentes para assumirem responsabilidades laborais e familiares. $\mathrm{Na}$ dimensão da "doença como causa de estigma e descriminação" (8 itens) os profissionais alcançaram valores entre 8 e 31 pontos, o que nos permite afirma que os inquiridos apresentam uma baixa crença pessoal de que a doença mental pode criar estigma e descriminação nos círculos íntimos dos doentes. os participantes tendem a discordar que a existência de doença mental cause embaraço ou vergonha, ou que os doentes devem ter receio de assumir o problema.

Na "perigosidade" os enfermeiros não vêm os doentes mentais como pessoas perigosas e com comportamentos imprevisíveis não acreditam que a doença mental leve à criminalidade ou que os portadores de doença mental sejam indivíduos sem competências sociais.

$\mathrm{Na}$ "condição médica", os participantes acreditam fortemente que a doença mental é uma condição clínica e, como outras doenças, precisam de tratamento medicamentoso na "responsabilidade individual", os enfermeiros não acreditam que cada individuo é responsável pela sua saúde mental ou que existe falta de cuidado das pessoas que sofrem desta tipologia de doença.

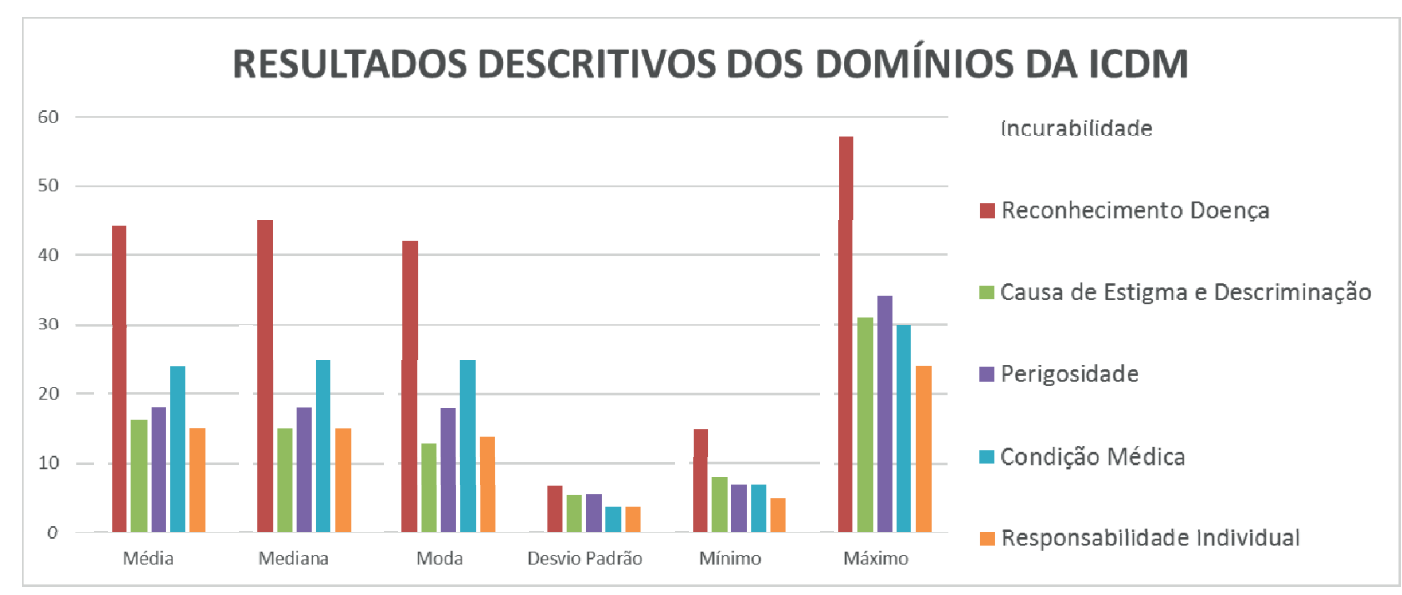

Gráfico 1 - Resultados Descritivos dos Domínios do ICDM 
$\mathrm{Na}$ dimensão "reconhecimento da doença" os inquiridos apresentam uma crença moderada alta não estigmatizante da doença mental e parece existir uma aceitação da doença e acreditação do tratamento e reabilitação dos doentes mentais desde que do início o tratamento seja adequado e atempado.

Transparece a ideia de que os inquiridos não manifestam crenças claramente negativas sobre a doença mental, tendo consciência de que a presença de uma doença mental não está relacionada com a sorte do individuo, mas que o tratamento passa essencialmente pelo cumprimento da terapêutica.

\section{Escala sobre as opiniões sobre a doença mental (EODM)}

Passando para os resultados da escala sobre as opiniões, o primeiro domínio, designado por Autoritarismo, os inquiridos obtiveram um score moderado acreditando que o doente mental tende a necessitar de intervenção coerciva por parte dos profissionais de saúde, pois tendem a apresentar um funcionamento algo estereotipado.

$\mathrm{Na}$ "benevolência" a média sugere que os participantes apresentam moderadamente uma atitude "simpática" para com o doente mental, porém um pouco paternalista e protetora. Apesar de encorajarem o tratamento, apresentam simultaneamente algum receio perante o doente, fundamentado na vulnerabilidade que reconhecem nestes doentes.
Quanto à "ideologia da doença mental", o que indica que os enfermeiros apresentam uma opinião moderada e neutra sobre a doença mental.

Existe resistência ao estigma tradicional e confiança na intervenção dos profissionais de saúde, mas ainda assim, o doente mental não é visto como uma pessoa completamente normal.

Relativamente à "restrição social" os inquiridos tendem a acreditar que as estruturas e contextos de tratamento devem apresentar alguma rigidez e restrição para o doente), apesar de não os considerarem mais perigosos que as pessoas em geral e de não se justificar o isolamento social e familiar.

Na dimensão da "etiologia interpessoal", a média indica que existe a tendência para os inquiridos não considerem a doença mental como resultado direto das escolhas individuais feitas ao longo da vida ou das circunstâncias da infância, embora a escolha e manutenção do tratamento dependa primordialmente do doente.

Apesar das opiniões não serem claramente negativas, estes resultados demonstram que os inquiridos acreditam que deve existir alguma imposição dos profissionais ao longo do tratamento, e que o doente é o principal responsável pelo seu tratamento e estado de saúde, mas que não deve estar restringido socialmente.

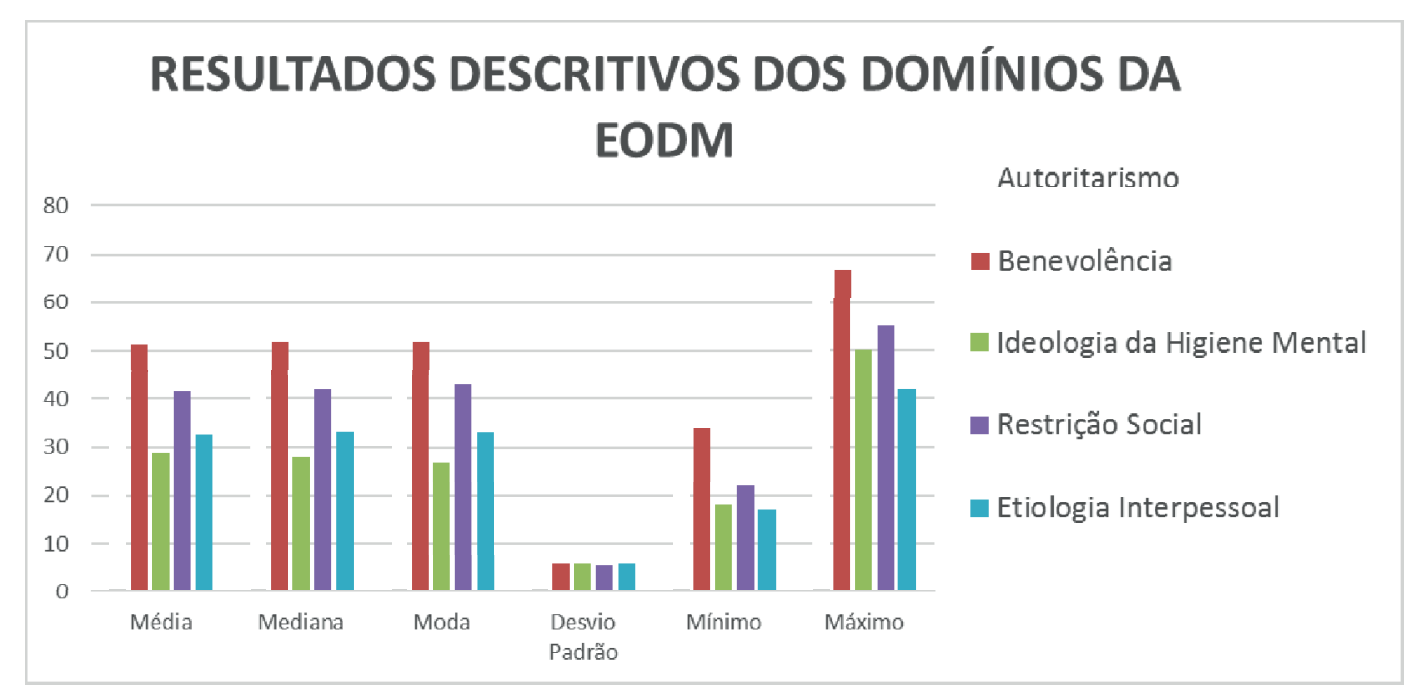

Gráfico 2 - Resultados Descritivos dos Domínios do ICDM 
Escala das Atitudes dos Clínicos (profissionais de saúde) (MICA-4)

No que se refere ao MICA-4, é importante relembrar que a pontuação mínima é de 16 e a máxima é de 96 , sendo que resultados mais baixos representam perceções menos estigmatizantes face à psiquiatria e à doença mental.
Os participantes do estudo alcançaram de 17 a 59 pontos, e a média está aproxima dos 32 valores que demonstra que o score é relativamente baixo dentro do intervalo possível de obter. reforçando a ideia que os profissionais não apresentam uma atitude crítica relativamente à doença mental, logo a presença de estigma é relativamente baixa.

\section{RESULTADOS DESCRITIVOS DO MICA-4}

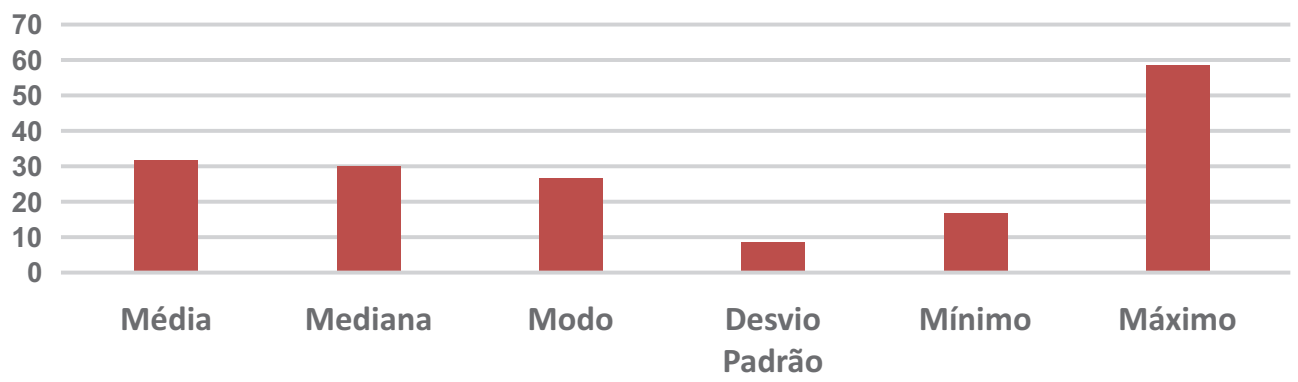

Gráfico 3 - Resultados Descritivos do MICA-4

\section{Discussão}

Numa análise mais cuidada, selecionamos estudos realizados por outros autores neste âmbito, embora com populações diferentes, e nos quais foram utilizados os mesmos instrumentos de colheita de dados: Escala de Opiniões acerca da Doença Mental (EODM); Inventário de Crenças sobre a doença Mental (ICDM; Escala das Atitudes dos Clínicos (MICA4), e tentamos perceber, se os resultados obtidos no nosso estudo e com a nossa população se afastam ou se aproximam dos resultados obtidos por esses estudos de outros autores

Analisando o Inventário de Crenças acerca da Doença Mental (ICDM) e numa comparação com resultados obtidos em outros estudos nomeadamente: com alunos de enfermagem (Gil, 2010); e com a população em geral (Loureiro 2008), os domínios do "reconhecimento da doença e crença da doença" como condição médica, foram as áreas, à semelhança dos resultados obtidos no nosso estudo, em que mais se evidenciou uma maior consciencialização por parte dos inquiridos, de que as perturbações psiquiátricas são de fato de "ordem médica" e que um diagnóstico precoce e tratamento atempado contribuem para uma melhoria significativa da qualidade de vida dos doentes e famílias (Loureiro,2008; Gil, 2010). Em contrapartida a crença de que a doença seja causa para estigma e descriminação, é o domínio que apresenta valores mais baixos.
A crença na "incurabilidade" e na "perigosidade" apresentam valores medianamente elevados. ${ }^{10}$

Os resultados obtidos no nosso estudo, nestas dimensões, os enfermeiros manifestam baixa crença pessoal de que a doença mental por si só poderá causar estigma e descriminação; não vêm os doentes mentais como pessoas perigosas e com comportamentos imprevisíveis; nem consideram que cada individuo seja responsável pela sua saúde mental acreditam fortemente que a doença mental é uma condição clinica, como o são outras patologias e que precisam de tratamento medicamentoso e psicoterapêutico

$\mathrm{Na}$ Escala de Atitudes sobre a doença Mental (EADM), que avalia as atitudes face à doença mental4, refere resultados muito semelhantes aos do nosso estudo, em que apresenta médias mais reduzidas nos domínios do "autoritarismo", "restrição social".

Sobre a ideologia da higiene mental, a população estudada por Loureiro, atribui como causas mais prováveis para o aparecimento da patologia psiquiátrica, "traumas emocionais e profissionais" além da deterioração física e envelhecimento. Segundo os autores, estes resultados manifestam alterações positivas na perceção que as pessoas têm sobre a doença mental.

Segundo o estudo de $\mathrm{Gil}^{11}$, as dimensões "benevolência" e "ideologia da higiene mental", apresentam 
médias mais altas, o que segundo esta autora poderá indicar maiores" níveis de tolerância e sensibilidade sociais" em relação a esta temática, associando os valores obtidos das atitudes mais positivas, perante os doentes mentais, à "relação de confiança na eficácia das intervenções dos profissionais especializados em saúde mental".

No que se referem às dimensões do "autoritarismo" e "restrição social", os inquiridos do nosso estudo, tendem a crer que o doente mental necessita de alguma intervenção coerciva por parte dos profissionais de saúde, por apresentarem um funcionamento algo estereotipado e acreditam que as estruturas e contextos de tratamento devem apresentar alguma rigidez e restrição para com o doente,

Comparando os resultados obtidos com os do estudo em que a população eram estudantes de enfermagem11 e contrariamente aos nossos resultados, verificamos que estas duas dimensões, são as que apresentam por parte dos inquiridos atitudes mais negativas, situação que a autora justifica, como estando associada à ausência de prática clinica por parte destes estudantes.

Relativamente ao MICA-4, os valores apresentados no nosso estudo, foram maioritariamente baixos o que nos permite afirmar que estes profissionais não apresentam atitudes críticas e negativas relativamente aos doentes mentais, sendo a presença de estigma relativamente baixo,

Com este instrumento, na avaliação das atitudes, não se verificaram diferenças estatísticas mediante o sexo, confirmando que esta variável não apresenta grande influência.

Comparamos os nossos resultados, com um estudo realizado por Queiroz, com alunos de medicina, em 2013, onde este instrumento foi aplicado, e onde não se encontraram igualmente diferenças significativas quanto ao género, idade ou características sociodemográficas dos participantes no que se refere à presença de estigma. $^{12}$

Salienta-se, no entanto, que a variável formação, apresenta diferenças, sendo que os alunos dos últimos anos do curso de medicina, apresentam mais atitudes estigmatizantes refentes à especialidade. Contrariamente aos resultados do nosso estudo que nos leva a concluir que os profissionais que mais formações procuram, são os que têm menos atitudes estigmatizantes.

\section{Limitações do estudo}

Quanto às limitações do estudo, as não respostas foram muitas, tendo sido manifestado com alguma frequência por parte destes profissionais, o argumento dos instrumentos de investigação serem extensos e morosos; exigirem uma maior concentração e foco na escolha das respostas; alem disso algumas questões em diferentes questionários, pareciam-lhes semelhantes.

Outra das razões que me verbalizaram, foi o fato de "estarem saturados de preencherem questionários" para os diferentes estudos, em diferentes temáticas, ou seja os pedidos de colaboração constantes para participação nos estudos de investigação de cariz académico, desmotiva-os.

O tempo estipulado para a realização do estudo, com todas as suas etapas, foi para nós, outra das limitações. Conseguir uma adesão fortemente significativa na colaboração do estudo por parte dos enfermeiros de saúde mental, ter respostas favoráveis das comissões de ética em tempo útil, e cumprir as restantes etapas, dentro dos prazos estipulados pela ESEP, não nos foi possível, obrigando-nos a solicitar adiamento da entrega da tese.

A demora por parte das Comissões de Ética das instituições em questão, em permitirem a realização do estudo, foi outra das limitações, atrasando a entrega dos questionários.

A pouca adesão por parte dos profissionais ao preenchimento dos questionários, condicionou a nossa amostra disponível e válida.

\section{Conclusão}

Em termos de conclusão, os resultados dos instrumentos utilizados, demonstram que existe pouca influência dos dados demográficos, pessoais e académicos nas crenças, opiniões e atitudes dos profissionais face à doença mental, no entanto, parece existir uma relação entre os três instrumentos, ou seja, a opinião e as crenças dos inquiridos sobre a doença mental parece ter uma relação direta com as atitudes e com a presença de estigma. 
Concluímos igualmente que a formação específica contribui como elemento protetor na redução das atitudes estigmatizantes,

Nos resultados da variável ter um familiar, ou amigo com patologia psiquiátrica, verificamos que não influenciam significativamente os resultados em nenhum dos instrumentos. No entanto os resultados diferem quando analisamos a variável: ter um colega com patologia psiquiátrica, sendo os valores de presença de estigma, ligeiramente mais elevados. Sendo assim, e atendendo as nossas questões de partida, obtivemos as seguintes respostas:

Com os resultados obtidos no nosso estudo, permitem-nos concluir que os enfermeiros não são agentes estigmatizantes, que a formação específica funciona com elementos protetor contra o estigma e que as nossas atitudes estão intimamente relacionadas com as nossas crenças.

\section{Contribuições para a enfermagem}

Considerando que as nossas opiniões, crenças e atitudes, condicionam os nossos comportamentos, e sendo estas, maioritariamente alicerçadas no desenvolvimento da nossa personalidade, seria importante, de uma forma adequada, "levar a doença mental", às faixas etárias mais jovens. Trabalhar a cidadania, a inclusão, a patologia psiquiatria, à semelhança de outros programas implementados nas escolas, sobre outras patologias, julgamos ser um bom princípio.

Pensamos que, se "incluirmos a doença mental" nas faixas etárias mais jovens, existe uma grande probabilidade de termos cidadãos melhor informados, mais inclusivos, e consequentemente, profissionais seja na área da saúde, seja em outras áreas, menos estigmatizantes. Pelo que verificamos anteriormente, a formação específica em Saúde Mental, funciona com fator protetor contra atitudes estigmatizante, pelo que é nossa sugestão, que cada instituição, investisse mais tempo e recursos, no debate aberto e construtivo entre profissionais sobre esta temática: Estigma.

Às instituições de ensino em saúde, sugere-se igualmente, a inclusão da temática estigma nos seus planos curriculares, seja estigma em doença mental, seja em outras áreas. Por si só, a palavra estigma já comporta uma conotação negativa, uma subjetividade quase sempre depreciativa. Faz sentido, que os futuros profissionais de saúde, sintam abertura e transparência para falarem e explorarem as suas emoções, crenças e consequentemente as suas atitudes, sem se sentirem constrangidos pelo simples fato de pensarem de forma diferente, ou mesmo por insegurança nos seus conhecimentos teóricos/práticos.

\section{Referências bibliográficas}

1. KRÜGER, Helmuth. Cognição, estereótipos e preconceitos sociais. Estereótipos, preconceitos e discriminação: perspectivas teóricas e metodológicas, 2004, 1: 23-40.

2. CAMPOS, Paula Manuela. Crenças, opiniões e atitudes dos enfermeiros de saúde mental, face à doença psiquiátrica. Master's Thesis, 2017.

3. VALA, Jorge. Representações sociais e psicologia social do conhecimento quotidiano. Psicologia social, 2000, 7: 457-502.

4. NEIVA, ER; MAURO, TG (2011). Atitudes e mudança de atitudes. In Torres \& Neiva (Orgs.), Psicologia social: Principais temas e vertentes, 2011, 171-20

5. FIRMIN, Ruth L., et al. Stigma resistance is positively associated with psychiatric and psychosocial outcomes: A meta-analysis. Schizophrenia Research, 2016, 175.1-3: 118-128.

6. MEHTA, Nisha, et al. Evidence for effective interventions to reduce mental health-related stigma and discrimination in the medium and long term: systematic review. The British Journal of Psychiatry, 2015, 207.5: 377-384.

7. GUIMARÃES, Cármen. Crenças dos profissionais de saúde acerca de determinantes de comportamentos de saúde: construção de um instrumento de avaliação. 2012. PhD Thesis.

8. KASSAM, A., et al. Development and responsiveness of a scale to measure clinicians' attitudes to people with mental illness (medical student version). Acta Psychiatrica Scandinavica, 2010, 122.2: 153-161.

9. GABBIDON, Jheanell, et al. Mental Illness: Clinicians' Attitudes (MICA) Scale-Psychometric properties of a version for healthcare students and professionals. Psychiatry research, 2013, 206.1:81-87.

10. DE JESUS LOUREIRO, Luís Manuel; AMARAL DIAS, Carlos Augusto; OLIVEIRA ARAGÃO, Rui. Crenças e Atitudes acerca das doenças e dos doentes mentais Contributos para o estudo das representações sociais da loucura. Referência-Revista de Enfermagem, 2008, 2.8.

11. GIL, Isabel Maria de Assunção. Crenças e atitudes dos estudantes de Enfermagem acerca das doenças e doentes mentais: Impacto do Ensino Clínico de Enfermagem de Saúde Mental e Psiquiatria. 2010. Master's Thesis.

12. QUEIROZ, Maria Inês Marques Araújo Carregal. Perceção dos alunos de medicina face à doença mental: estudo exploratório sobre diferenças de género. 2013. PhD Thesis. 